\title{
Integration optimization, triage and analysis tools for serial crystallography
}

\author{
Artem Y. Lyubimov, Monarin Uervirojnangkoorn, Aina E. Cohen and Axel T. Brunger
}

Serial crystallography (SX), whereby still (zero-rotation) diffraction images are collected from multiple crystals at randomized orientations, is rapidly emerging as a useful tool for structural analysis of radiation-sensitive samples at microsecond (synchrotron) or femtosecond (XFELs) scale. As SX is rapidly becoming accessible to the broader crystallographic community, a need has developed for versatile, powerful, and userfriendly data processing software. The integration optimization, triage and analysis (IOTA) toolkit serves as a front-end for both cctbx.xfel or DIALS, with integration optimization tools available for the former and in development for the latter. Pairing the optimization tools in IOTA with the post-refinement and merging program PRIME, researchers can obtain interpretable merged datasets from very limited $(<1000)$ amounts of diffraction images, making this technique most suitable for projects where crystalline material is in limited supply. The rapid, easy-to-read graphical feedback allows users to make on-the-spot adjustments to experimental parameters, thus increasing the success of high-rate SX experiments. 